\title{
Influence of Molecular Design on Radical Spin Multiplicity: Characterisation of BODIPY Dyad and Triad Radical Anions.
}

Barry Mangham, ${ }^{1}$ Magnus W. D. Hanson-Heine, ${ }^{1}$ E. Stephen Davies, ${ }^{1}$ Alisdair Wriglesworth, ${ }^{1}$ Michael W. George, ${ }^{1,2}$ William Lewis, ${ }^{1}$ Deborah L. Kays, ${ }^{1}$ Jonathan McMaster, ${ }^{1}$ Nicholas A. Besley, ${ }^{1}$ and Neil R. Champness ${ }^{1 *}$

${ }^{1}$ School of Chemistry, University of Nottingham, University Park, NG7 2RD, U.K.

${ }^{2}$ Department of Chemical and Environmental Engineering, University of Nottingham Ningbo China, 199 Taikang East Road, Ningbo 315100, China

A strategy to create organic molecules with high degrees of radical spin multiplicity is reported in which molecular design is correlated with the behaviour of radical anions in a series of $B O D I P Y$ dyads. Upon reduction of each BODIPY moiety radical anions are formed which are shown to have different spin multiplicities by electron paramagnetic resonance (EPR) spectroscopy and distinct profiles in their cyclic voltammograms and UV-visible spectra. The relationship between structure and multiplicity is demonstrated showing that the balance between singlet, biradical or triplet states in the dyads depends on relative orientation and connectivity of the BODIPY groups. The strategy is applied to the synthesis of a BODIPY triad which adopts an unusual quartet state upon reduction to its radical trianion.

The synthesis of organic radicals has received extensive focus over recent years with diverse interests in areas including redox catalysis, imaging, magnetochemistry and molecular spintronics. ${ }^{1-3} \mathrm{~A}$ wide variety of organic moieties are suitable for the creation of stable multicentred radical species, these include phenothiazine, ${ }^{4}$ nitroxide, ${ }^{5,6}$ aminyl, ${ }^{7}$ viologen, ${ }^{8}$ dithiazolyl, ${ }^{9}$ verdazyl, ${ }^{10}$ and macrocyclic polyradicaloids. ${ }^{11}$ Many of these systems exists as triplet states although examples of higher spin multiplicities are known but are significantly less common. Such higher spin multiplicities require strong coupling between the spins, typically as a result of a defined arrangement and this approach has led to the identification of stable quartet ${ }^{12-17}$ and quintet ${ }^{18,19}$ states in multi-centred organic radicals.

In parallel main group elements, ${ }^{20,21}$ including boron, have been incorporated into free radical manifolds and this strategy has led us to investigate the use of BODIPY (4,4-difluoro4-bora-3a,4a-diaza-s-indacene) compounds to create radical species. Our previous investigations of BODIPY molecules ${ }^{22}$ reveal that the electrochemical reduction of the BODIPY core leads to the formation of a radical anion (BODIPY*-) which can be characterized by electron paramagnetic resonance (EPR) spectroscopy. BODIPY compounds have been developed for many applications, predominantly based on their fluorescent properties, and as a result the synthetic approaches to functionalised BODIPY species are advanced. ${ }^{23-25}$ We anticipated that if molecules with multiple BODIPY components could be found which exhibited spin coupling, leading to higher or unusual spin multiplicities, then the rich chemistry of BODIPY compounds would allow the development of new families of organic radicals.

Synthetic strategies for the formation of BODIPY dyads ${ }^{26,27}$ and a small number of triads $^{28}$ with various geometries have been reported. Thus, we decided to explore, for the first time, the behavior of reduced BODIPY dyads and triads and in particular the multiplicity of these radical anion species. In this study we report a series of five BODIPY dyads and a triad in which the relative geometry and spacing of the BODIPY cores is systematically controlled 
(Fig. 1a). We then studied the electrochemical behavior of these systems and probed the behavior of the di-reduced $\left([1]^{2-},[2]^{2-},[4]^{2-},[5]^{2-},[6]^{2-}\right)$, or tri-reduced $\left([3]^{3-}\right)$, states in order to correlate structure with multiplicity. Our experimental observations, supported by density functional theory (DFT) and second-order multireference perturbation theory calculations, indicate the formation of singlet, open-shell singlet, triplet and quartet states depends on the molecular architecture.

\section{Results and Discussion}

Dipyrromethane precursors for all five BODIPY dyads (1, 2, 4-6) and triad (3) were synthesised according to a modified literature procedure (see SI for details). ${ }^{29}$ In all instances a di-aldehyde, or tri-aldehyde for $\mathbf{3}$, were condensed with pyrrole using trifluoroacetic acid as catalyst to afford the target dipyrromethanes or tripyrromethane in $70-90 \%$ yield with the exception of the durene-bridged dipyrromethane which was isolated in $20 \%$ yield. Target BODIPY species were prepared using a modified literature procedure. ${ }^{30}$ Dipyrromethanes were oxidised using 2,3-dichloro-5,6-dicyano-1,4-benzoquinone (DDQ) and subsequently reacted with $\mathrm{BF}_{3} . \mathrm{OEt}_{2}$ in the presence of a base, $\mathrm{N}, \mathrm{N}$-diisopropylethylamine (DIPEA). In all cases low yields were observed for the BODIPY formation reactions which was attributed to the decomposition of oxidised dipyrromethane species through polymerisation of pyrrole sub units at the alpha position, ${ }^{31}$ and observed though the formation of a black polymeric residue. It was noted that oxidation time and reaction solvent affected yields significantly. Indeed, shortening the reaction time for the oxidation step from 1 hour to less than 5 minutes resulted in increased yields, $c a$. $5-10 \%$ from less than $1 \%$. Furthermore, yields were improved by using anhydrous toluene as solvent rather than $\mathrm{CH}_{2} \mathrm{Cl}_{2}$. Similar syntheses of $\mathbf{1}$ and $\mathbf{2}$ in similar yield have been reported previously using $\mathrm{InCl}_{3}$ as catalyst. ${ }^{26}$ It should be noted that alternative synthetic strategies have been used to prepare related BODIPY dyad and triad systems in slightly higher yields replacing the aldehyde precursor with the corresponding acyl chloride. ${ }^{28}$

The single crystal structures of $\mathbf{1}, \mathbf{2}$ and $\mathbf{3}$ were determined from crystals grown by the slow diffusion of $\mathrm{MeOH}$ into solutions of the compounds in $\mathrm{CH}_{2} \mathrm{Cl}_{2}$. Attempts to grow single crystals of 4-6 using analogous conditions were unsuccessful. The structures of $\mathbf{1}$ and $\mathbf{2}$ have been reported previously ${ }^{26}$ and our measurements confirm similar structural arrangements. In 1 the BODIPY moieties are found to be co-planar but sit at an angle of $54.1^{\circ}$ with respect to the phenyl linker. In contrast to $\mathbf{1}$, the BODIPY moieties in $\mathbf{2}$ are offset, in a propeller conformation with respect to each other across the phenyl linker and at an angle of $52.1^{\circ}$ with respect to the phenyl linker. $\mathbf{3}$ adopts a similar arrangement to $\mathbf{2}$, with the BODIPY moieties arranged in a propeller configuration across the central phenyl linker (Fig. 1b). The relative orientation of the BODIPY units with respect to each other and to the linking unit in each compound is notable as this may influence communication through the molecule but it should be noted that crystal structures are not necessarily indicative of solution phase behaviour.

All six compounds exhibited similar fluorescence, typical of BODIPY species functionalised at the meso-position with aryl groups. ${ }^{22,27,28}$ Spectra exhibited small Stoke's shifts ( 20-30 nm) and quantum yields less than 0.03 in all cases except for compound 4. 4 exhibited the smallest Stoke's shift, $18 \mathrm{~nm}$, and the longest fluorescence lifetime of $9 \mathrm{~ns}$ and this was considered to result from the inhibition of free rotation at the meso position in 4 compared to others in the series. UV-visible spectroscopy is discussed in more detail below, particularly in comparison to reduced radical species. 
a)
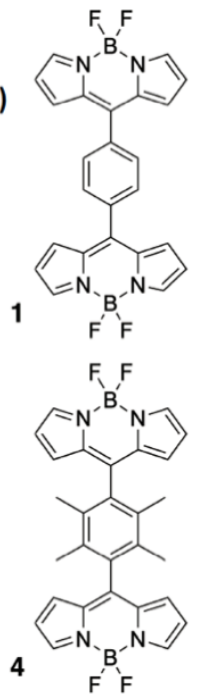
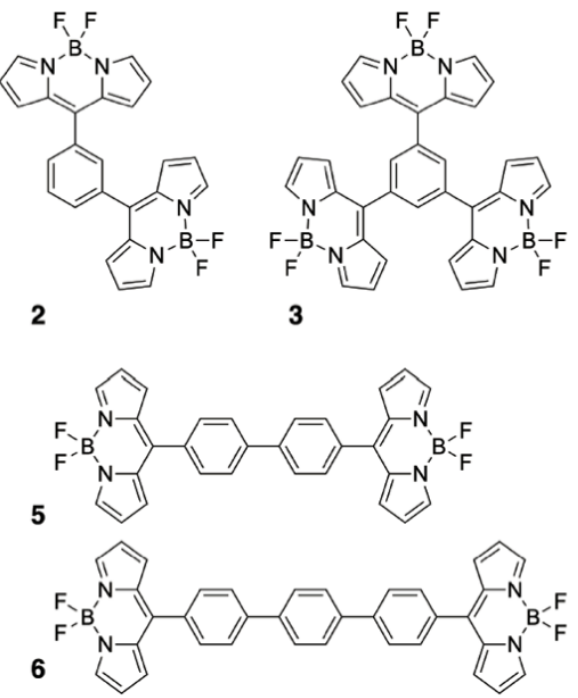

b)
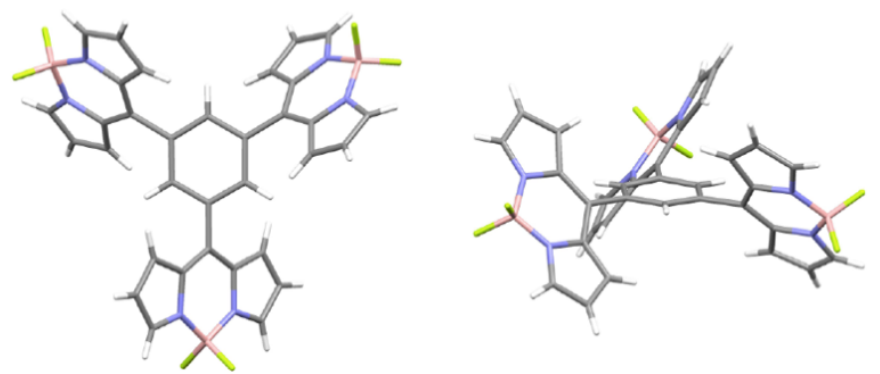

Fig. 1. a) BODIPY dyads and triad reported herein; b) views of the single crystal X-ray structures of 3. Colour guide: boron (pink), fluorine (yellow), nitrogen (blue), carbon (grey), hydrogen (white).

In order to probe the nature of the reduced states for 1-6, cyclic and square wave voltammetry studies were conducted at room temperature in $\mathrm{CH}_{2} \mathrm{Cl}_{2}$ with $\left[{ }^{n} \mathrm{Bu}_{4} \mathrm{~N}\right]\left[\mathrm{BF}_{4}\right]$ as supporting electrolyte. In each case reversible reduction processes are observed for the compounds as anticipated for phenyl-substituted BODIPY moieties. ${ }^{22,26}$ Whilst each BODIPY unit is expected to undergo a single one-electron reduction the number of reduction processes observed for 1-6 varies depending upon the geometry and length of the linker, and more particularly the substitution of the linker unit which is affecting communication between the redox centres (Table S1). Thus, whilst the para substituted dyad, $\mathbf{1}$, displayed a single well resolved reduction $\left(E_{1 / 2}=-1.03 \mathrm{~V}\right.$ ) that showed scan rate dependence (Fig. 2a), the meta-substituted dyad, 2, displayed two overlapping reductions in the cyclic voltammetry $\left(E_{1 / 2}=-1.10,-1.22 \mathrm{~V}\right)$ (Fig. $\left.2 \mathrm{~b}\right)$ and the triad, 3, displayed three overlapping reductions $\left(\mathrm{E}_{1 / 2}=\right.$ $-1.02,-1.14,-1.27 \mathrm{~V})$ (Fig. 2c). Where more than one process was observed potentials were resolved by square wave voltammetry. The observations are consistent with 1 undergoing a single, two-electron reduction with attractive interactions between the added electrons. ${ }^{32,33}$ 2 exhibits two successive one electron reductions separated by $0.12 \mathrm{~V}$, and $\mathbf{3}$ undergoes three successive one electron reductions, separated by 0.11 and $0.13 \mathrm{~V}$. For both 2 and 3 the separation between the reduction processes suggests limited communication involving repulsive (Coulombic) interactions between added electrons. The cyclic voltammograms for $\mathbf{1}$ and $\mathbf{2}$ have been reported previously ${ }^{26}$ and our experimental data are in good agreement with the previous study. Interestingly, studies of $\mathbf{4}$ revealed two closely overlapping reversible reductions which were resolved by square wave voltammetry $(-1.22,-1.31 \mathrm{~V})$ (Fig. $2 \mathrm{~d})$. The 
separation of the two reduction processes, in contrast to the single reduction associated with $\mathbf{1}$, is indicative of repulsive interactions between electrons located on the two terminal BODIPY moieties in 4. Cyclic voltammetry studies of 5 and 6 both revealed single, well resolved, reductions $\left(5: E_{1 / 2}=-1.17 \mathrm{~V} ; 6: E_{1 / 2}=-1.16 \mathrm{~V}\right.$ ) involving two electrons, similar to the behaviour of $\mathbf{1}$, but at more negative potentials, consistent with greater electron density donation to the BODIPY centres by the bi- or ter-phenyl linkers. The reduction potentials for 5 and 6 are consistent with that of $N, N^{\prime}$-difluoroboryl-5-(phenyl)dipyrrin $\left(E_{1 / 2}=-1.19 \mathrm{~V}\right)$ which contains a single BODIPY unit and a phenyl group on the dipyrrin meso-carbon and indicate that an acceptor orbital of similar nature and energy is common to these species. ${ }^{22}$ The shift to less negative for the reduction of 1 is consistent with the electrons entering a more stabilised molecular orbital than those found in $\mathbf{5}$ and $\mathbf{6}$ (see below).

Whilst the cyclic voltammetric profiles of $\mathbf{1 , 5}$ and $\mathbf{6}$ all appear similar, analysis of the effect of scan rate on the peak separations $\left(\Delta E=E_{p}{ }^{a}-E_{p}{ }^{c}\right)$ suggests that upon reduction the BODIPY moieties interact differently in each case. Interactions in $\mathbf{1}$, as discussed above, show a decrease in peak separation at slower scan rates indicating a two-electron process involving attractive interactions, likely resulting from significant structural change, such as a rearrangement or a large change in solvation upon reduction. For $\mathbf{5}$ the peak separations are consistently larger than expected and show little dependence on scan rate relative to that observed for ferrocene under these conditions (Table S2). This suggests a regime in which weak coulombic interactions exist between the added electrons but unlike 2-4 these are insufficient to result in the resolution of two separate reduction waves, i.e. communication between the redox orbitals is present but weak. Extending the linker from bi-phenyl in $\mathbf{5}$ to ter-phenyl in 6 increases the distance between redox centres and further decreases the extent of interaction (Figs S3, S4). 6 shows peak separations that are comparable to those of ferrocene suggesting that this increase in distance between redox centres results in little interaction between the added electrons.

a)

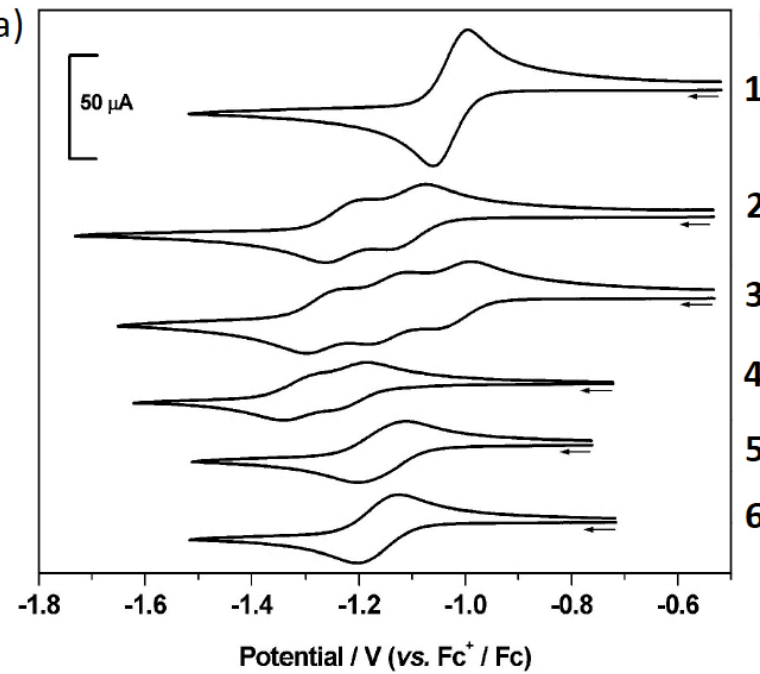

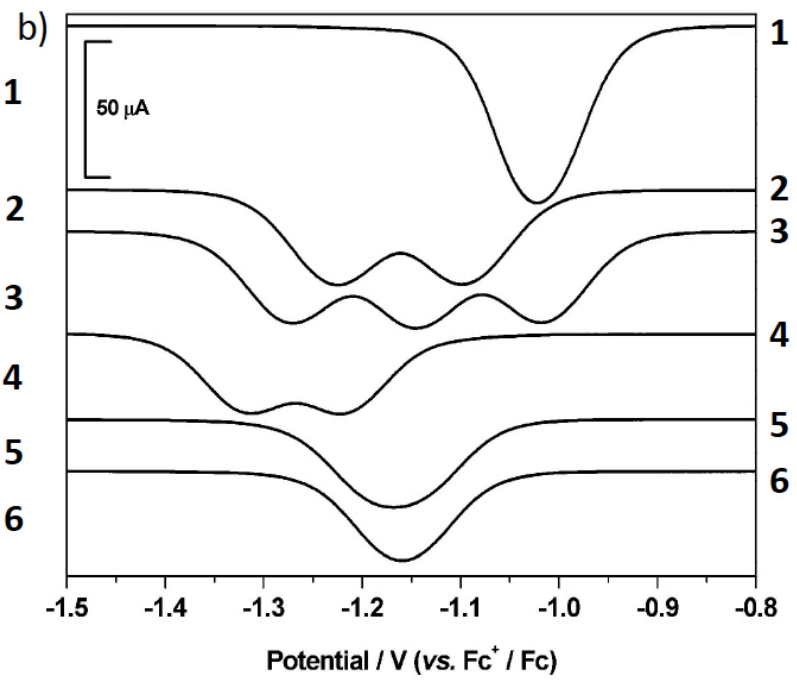

Fig. 2. a) Cyclic voltammograms of 1-6 recorded at $\mathrm{RT}$ in $\mathrm{CH}_{2} \mathrm{Cl}_{2}$ with $\left[{ }^{n} \mathrm{Bu}_{4} \mathrm{~N}\right]\left[\mathrm{BF}_{4}\right](0.4 \mathrm{M})$ as supporting electrolyte; b) square wave voltammetry indicating multiple independent but overlapping reduction processes for $\mathbf{2}, \mathbf{3}$ and $\mathbf{4}$.

The nature of the reduced, radical species was probed further by EPR spectroscopy in order to evaluate the influence of molecular structure, and in particular the geometry and length of the spacer separating the BODIPY moieties, upon the spin multiplicity of the radical species (Table 1). The results illustrate that the nature and arrangement of the spacer has a 
significant effect upon the EPR spectra observed for the different species. All radical dianionic, or trianionic, species gave EPR signals in both fluid and frozen EPR samples. In all cases fluid EPR spectroscopy showed a single resonance (Fig S5) at $g_{\text {iso }}=2.0026-2.0028$, however, analysis of frozen samples gave more complex spectra in a number of cases (Fig 3).

Table 1. Summary of EPR data for radical species of reduced forms of 1-6 at $77 \mathrm{~K}$ in $\mathrm{CH}_{2} \mathrm{Cl}_{2}$ with $\left[{ }^{\mathrm{n}} \mathrm{Bu}_{4} \mathrm{~N}\right]\left[\mathrm{BF}_{4}\right](0.4 \mathrm{M})$.

\begin{tabular}{|l|l|l|l|l|l|}
\hline Compound & \multicolumn{1}{|c|}{$\begin{array}{c}\mathrm{g}_{\text {iso }} \\
298 \mathrm{~K}\end{array}$} & \multicolumn{1}{c|}{\begin{tabular}{c}
\multicolumn{1}{c|}{$\mathrm{g}^{\mathrm{a}}$} \\
$77 \mathrm{~K}$
\end{tabular}} & $\begin{array}{c}\text { Half-field } \\
\text { signal }\end{array}$ & \multicolumn{1}{c|}{$\mathrm{D} / \mathrm{cm}^{-1}$} & Assignment (77K) \\
\hline$[1]^{2-}$ & $-^{\mathrm{b}}$ & $-\mathrm{b}$ & - & - & Closed shell singlet \\
\hline$[2]^{2-}$ & 2.0028 & $2.0032(12)^{\mathrm{c}}$ & 4.0084 & $71 \times 10^{-4}$ & triplet \\
\hline$[3]^{3-}$ & 2.0026 & $2.0030(10)$ & 4.0084 & $39 \times 10^{-4}$ & quartet \\
\hline$[4]^{2-}$ & 2.0028 & $2.0030(13)^{\mathrm{c}}$ & 4.0086 & $82 \times 10^{-4}$ & triplet \\
\hline$[5]^{2-}$ & 2.0026 & $2.0029(15)$ & - & - & open-shell singlet \\
\cline { 2 - 7 } & - & $2.0029(15)$ & $\mathrm{d}$ & $50 \times 10^{-4}$ & triplet \\
\hline$[6]^{2-}$ & 2.0027 & $2.0025(12-$ & - & $15 \times 10^{-4}$ & triplet \\
\hline
\end{tabular}

${ }^{a}$ brackets show linewidth used in simulation, in Gauss; all simulations were generated using Gaussian lineshapes; ${ }^{b}$ weak signal observed, see text for discussion; ${ }^{c}$ additional signal at $g=$ 2.0028 consistent with signal observed at ambient temperature; ${ }^{d}$ not observed.

Interesting contrasts are observed between the various radicals with spin multiplicity clearly affected by the geometrical arrangement of the spacer (Fig. 3). The EPR spectrum of $[1]^{2-}$, gives rise to a weak signal in both fluid and frozen samples. When integrated against the stable radical DPPH under the same conditions the observed spectrum represents less than 2 $\%$ of the expected spin. Therefore, the observed spectrum that arises from the reduction of 1 is considered to arise from a small amount of a paramagnetic impurity and $[1]^{2-}$ exists in the EPR-silent closed-shell singlet state. In contrast, the spectrum observed for [2] ${ }^{2-}$ is consistent with an open-shell singlet diradicaloid as a fluid solution but on cooling to $77 \mathrm{~K}$ additional features are resolved in the full-field $(g \approx 2)$ and half-field $(g \approx 4)$ regions consistent with a triplet species. The triplet nature of the frozen EPR spectrum of [2] $]^{2-}$ was confirmed by simulation of the observed four line spectrum in the $g=2$ region using a zero field splitting energy ( $|\mathrm{D} / \mathrm{hc}|)$ of $71 \times 10^{-4} \mathrm{~cm}^{-1}$ and axial geometry (i.e. $\mathrm{E}=0$ ) for the $\Delta \mathrm{M}_{\mathrm{s}}= \pm 1$ transitions. The presence of a weak half-field signal $(g=4.008)$, corresponding to the forbidden $\Delta M_{s}= \pm 2$ transition, helped confirm our assignment of [2] ${ }^{2-}$ as a triplet radical (Fig 3b). The feature in the centre of the spectrum, not reproduced by the simulation, most likely arises due to a paramagnetic by-product, such features are often reported in studies of biradical species. ${ }^{34,35}$ Differences between isomers [1] ${ }^{2-}$ and $[2]^{2-}$ indicate that the arrangement of the BODIPY moieties, para vs. meta, is critical in determining the spin multiplicity of the radical anions. The effect of geometry upon EPR multiplicity is further demonstrated by the spectrum of a frozen sample of $[3]^{3-}$ which shows five lines at X-band frequency (Fig. $3 \mathrm{c}$ ) together with a signal at half field, consistent with, and simulated as, a quartet state with $\mathrm{g}=2.0030$ and $|\mathrm{D} / \mathrm{hc}|=39 \times 10^{-4} \mathrm{~cm}^{-1}$.

The effects of spacer orientation and length on spin multiplicity are demonstrated by considering the series of para-substituted compounds $[\mathbf{1}]^{2-},[4]^{2-},[5]^{2-}$ and $[6]^{2-}$. The BODIPY centres in [1] $]^{2-}$ and $[4]^{2-}$ are each separated by an aromatic 6 -membered ring, phenyl in the case of 1 and duryl, 2,3,5,6-tetramethyl-phenyl, in $\mathbf{4}$. In contrast to [1] ${ }^{2-}$ (see above) [4] $]^{2-}$ gives a triplet spectrum at $77 \mathrm{~K}$ (Fig. $3 \mathrm{~d}$ ). This illustrates the influence of conformation, not distance, 
on magnetic properties since the duryl spacer restricts rotation with respect to the BODIPY moieties. The longer fluorescence lifetime observed for $\mathbf{4}$ is perhaps indicative of this since hindered rotation inhibits non-radiative decay processes. ${ }^{36}$

Comparison of $[1]^{2-}$ with $[5]^{2-}$ and $[6]^{2-}$ probes the effect of increasing the separation between the two terminal BODIPY moieties by increasing the length of the spacer from phenyl $[1]^{2-}$, to biphenyl, $[5]^{2-}$, to terphenyl, $[6]^{2-}$. Strong coupling was observed between electrons in $[1]^{2-}$ to produce a closed-shell singlet state but cyclic voltammetry, as discussed above, shows that subtle differences exist in the electronic interactions for the dianions in this series. As anticipated, larger separation between the BODIPY moieties, on which reduction is predominantly based, leads to weaker interactions between electron spins in $[5]^{2-}$ and $[6]^{2-}$ than those in $[\mathbf{1}]^{2-}$. Thus, whereas $[\mathbf{1}]^{2-}$ adopts a closed-shell singlet state $[\mathbf{5}]^{2-}$ and $[\mathbf{6}]^{2-}$ exhibit more complex behaviour. The frozen solution EPR spectrum of [5] ${ }^{2-}$ is more complex (Fig. $3 \mathrm{~d}$ ). Five lines are observed at full field but no half-field signal was detected. We attempted the simulation of this spectrum using individual components from an open-shell singlet diradicaloid (single resonance at centre of the spectrum) and a triplet (four lines) which leads to a good match. This result suggests more complex spin behaviour for [5] $]^{2-}$ although we note that a decomposition product with doublet character, as observed for $[\mathbf{1}]^{2-},[\mathbf{2}]^{2-}$ and $[4]^{2-}$, may contribute to the EPR spectrum but would appear unusually large if this were the sole origin of the signal. The possibility of both open-shell singlet diradicaloid and triplet states being observed for the same molecule may arise due to subtle changes in conformers in the frozen solution. ${ }^{37}$ In contrast to $[5]^{2-},[6]^{2-}$ gives a frozen solution EPR spectrum consisting of what initially appears to be a single resonance (Fig. 3e). However, modelling of this spectrum indicates triplet character at $77 \mathrm{~K}$, although we cannot rule out the presence of a minor component of open-shell singlet diradicaloid character.

In order to understand the behaviour observed for the radical anions we performed DFT calculations (see SI for computational details). The magnetic behaviour is best interpreted through comparison of compounds $\mathbf{1 , 2}$ and $\mathbf{3}$. The molecular orbitals of the neutral species, 1, were calculated and reveal that the highest four occupied orbitals comprise of two pairs of degenerate orbitals which correspond to in-phase and out-of-phase combinations of $\pi$ molecular orbitals on the BODIPY moieties (Fig 4a). The two lowest unoccupied orbitals are the $\pi^{*}$ orbitals of the BODIPY moieties and the next two are $\pi^{*}$ orbitals associated with the central benzene ring linker with a HOMO-LUMO energy gap of 0.247 a.u. Addition of two electrons to the molecule can result in singlet, open-shell singlet or triplet states. In our discussion singlet refers to the lowest energy closed-shell (all electrons paired) singlet state unless specified otherwise. DFT is a single reference determinant-based method and is not suited to describe open-shell singlet states which have multi-determinant character. In order to determine the relative energy of the open-shell singlet states relative to the singlet and triplet states second-order multireference perturbation theory calculations have also been performed (see SI for details). For [1] $]^{2-}$ the calculations confirm the singlet state to be lower in energy than the triplet state (Table 2). This is consistent with the orbital energy diagram for the neutral form of $\mathbf{1}$ as there is a reasonably large energy difference between the LUMO and LUMO+1 orbitals ( 0.013 a.u.), thus, the LUMO+1 orbital is less accessible. The orbital energy diagram for the singlet state of $[1]^{2-}$ (Fig. 4b) confirms that the LUMO from the neutral molecule is now doubly occupied giving rise to a singlet state. 

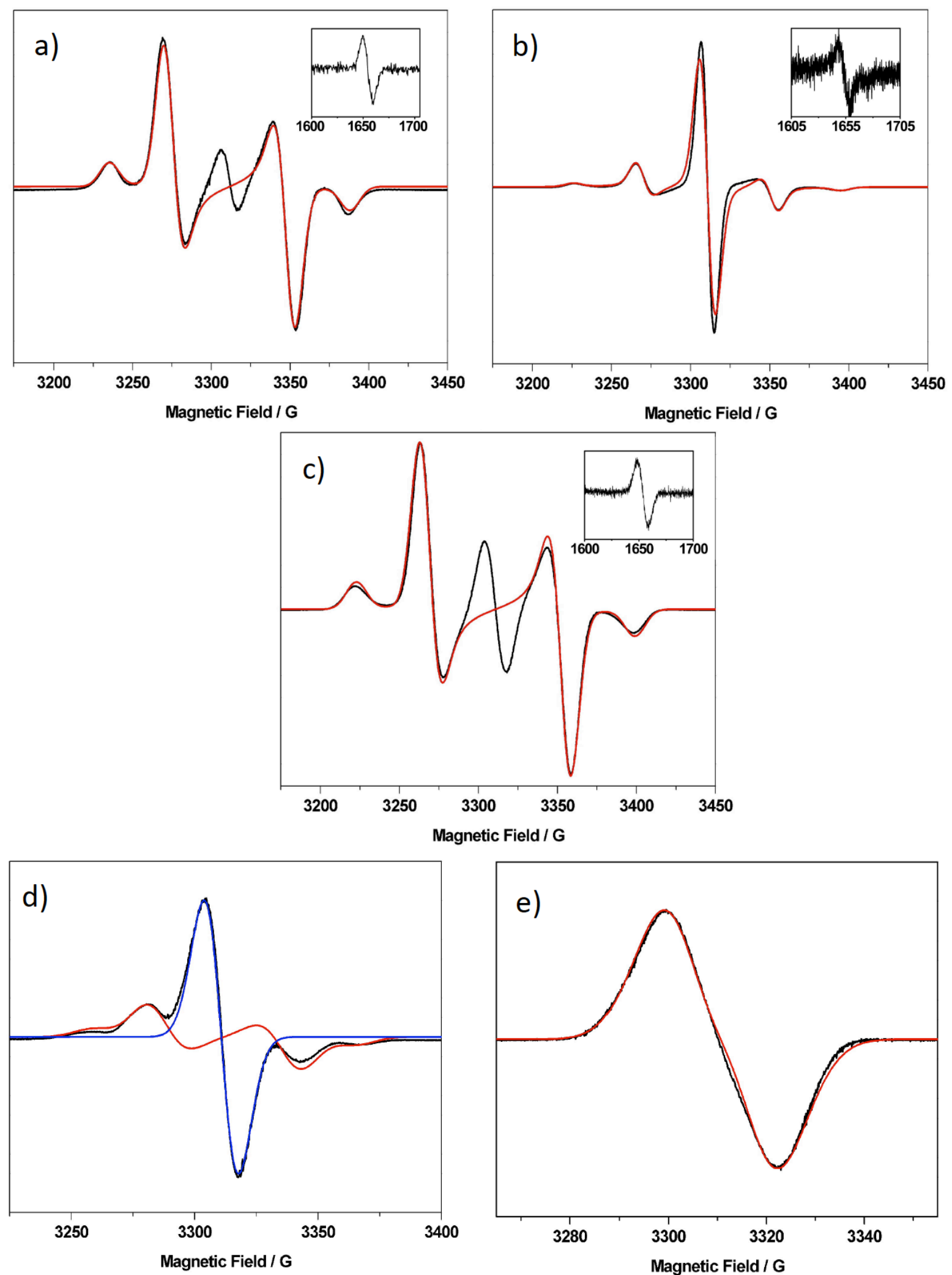

Fig. 3. EPR spectra a) $[2]^{2-}$, b) $[3]^{2-}$; c) $[4]^{3-}$; d) $[5]^{2-}$; e) $[6]^{2-}$ at $77 \mathrm{~K}$ in $\mathrm{CH}_{2} \mathrm{Cl}_{2}$ with $\left[{ }^{n} \mathrm{Bu}_{4} \mathrm{~N}\right]\left[\mathrm{BF}_{4}\right]$ $(0.4 \mathrm{M})$. Experimental data are shown in black. Simulations of doublet states are shown in blue, whereas simulations of triplet states (or a quartet state in the case of [3] ${ }^{3-}$ ) are shown in red. For a) and c) the un-simulated feature at the centre of the spectrum was thought to arise from a small quantity of doublet impurity. 
2 exhibits a similar pattern to that of $\mathbf{1}$ with a similar HOMO-LUMO energy gap (Fig $5 a)$, although for $\mathbf{2}$ the orbitals correspond to $\pi$ orbitals localised on a single BODIPY moiety. As with 1, addition of two electrons can give rise to singlet or triplet states but in the case of 2 the triplet state is the lowest in energy (Table 2). This can be partially rationalised by the smaller energy difference between the LUMO and LUMO+1 orbitals for 2 (0.003 a.u.) in comparison to 1 (0.013 a.u.). Therefore, the additional stability associated with the parallel spin electrons compensates for an electron lying in a slightly higher energy orbital. Analysis of the MOs for [2] ${ }^{2-}$ shows that the orbitals associated with the BODIPY moieties are singly occupied (Fig. 5b) whereas the LUMO orbitals in the triplet dianion are associated with the $\pi$ orbitals of the $m$-phenyl linker giving rise to a larger HOMO-LUMO gap (Table 2). The lower energy of the triplet state for [2] $]^{2-}$ is consistent with the observed EPR spectrum (Fig. 3b).

a)
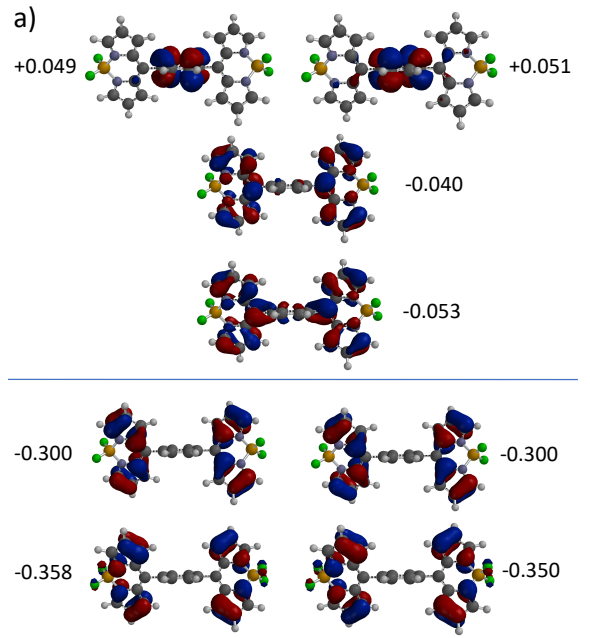

b)
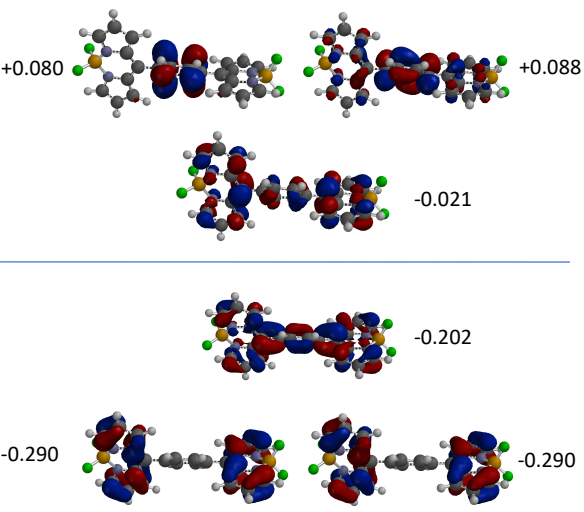

Fig. 4. DFT computed MO diagram for a) 1 in neutral ground state; b) the singlet form of [1] ${ }^{2-}$ with the energies of the molecular orbitals in atomic units. The line represents boundary between occupied and unoccupied orbitals.

Table 2. Calculated lowest energy states and HOMO-LUMO energy gaps for neutral ground state and closed shell singlet and open-shell high and low spin dianion compounds. The low spin state is a singlet for compounds $\mathbf{1}, \mathbf{2}, \mathbf{4 - 6}$ and doublet for $\mathbf{3}$, and the high spin state is a triplet for compounds 1, 2, 4-6 and quartet for 3.

\begin{tabular}{|c|c|c|c|c|c|}
\hline Compound & $\begin{array}{c}\text { Predicted } \\
\text { lowest energy } \\
\text { state for } \\
\text { radical anions }\end{array}$ & Neutral & $\begin{array}{c}\text { HOMO-LUMO energy gap (a.u.) } \\
\text { [compound] }^{2-(3-)} \\
\text { Closed-shell } \\
\text { singlet }\end{array}$ & $\begin{array}{c}\text { [compound }]^{2-(3-)} \\
\text { Open-shell low }^{\text {spin }}\end{array}$ & $\begin{array}{c}\text { [compound] }^{2-(3-)} \\
\text { Open-shell high } \\
\text { spin }\end{array}$ \\
\hline $\mathbf{1}$ & singlet & 0.247 & 0.181 & 0.204 & 0.265 \\
\hline $\mathbf{2}$ & triplet & 0.252 & 0.124 & 0.219 & 0.273 \\
\hline $\mathbf{3}$ & quartet & 0.250 & - & 0.207 & 0.274 \\
\hline $\mathbf{4}$ & triplet & 0.255 & 0.137 & 0.205 & 0.278 \\
\hline $\mathbf{5}$ & $\begin{array}{c}\text { open-shell } \\
\text { singlet }\end{array}$ & 0.251 & 0.143 & 0.038 & 0.249 \\
\hline $\mathbf{6}$ & $\begin{array}{c}\text { open-shell } \\
\text { singlet }\end{array}$ & 0.253 & 0.108 & 0.031 & 0.228 \\
\hline
\end{tabular}

As 3 undergoes a three-electron reduction process, neither a singlet or triplet state is accessible for $[3]^{3-}$. Calculations were performed to determine whether a doublet or quartet 
state is favourable. Similar to $\mathbf{1}$ and $\mathbf{2}$, the highest molecular orbitals calculated for the neutral form of $\mathbf{3}$ are energetically close combinations of $\pi$ molecular orbitals located on the BODIPY moieties. For 3 these three orbitals constitute the HOMO, HOMO-1 and HOMO-2 orbitals (see $\mathrm{SI})$. The three lowest unoccupied virtual orbitals are made up of energetically close combinations of $\pi^{*}$ molecular orbitals located on the BODIPY moieties. Addition of three electrons gives [3] $]^{3-}$ which can adopt either a doublet or quartet state, the energies for which suggest that the quartet state is more favourable as the ground state for the trianion.

4 differs from 1 in that the central linking phenyl ring in 1 is replaced by a duryl moiety. Similarly to 1, DFT calculations for 4 confirm that the four highest occupied molecular orbitals comprise of the almost degenerate in-phase and out-of-phase combinations of $\pi$ molecular orbitals on the BODIPY moieties which make up the HOMO and HOMO-1 orbitals (see $\mathrm{SI}$ ). There are also two $\pi$ orbitals located on the duryl linker making up the HOMO-2 and HOMO3 orbitals. The four lowest unoccupied virtual orbitals are analogous to those observed for 1 . The presence of tetramethyl substitution on the central phenyl ring gives a calculated HOMOLUMO energy gap of 0.114 a.u., larger than that calculated for either $\mathbf{1}$ or $\mathbf{2}$. In contrast to $[1]^{2-}$, the DFT and multireference perturbation theory calculations for $[4]^{2-}$ indicate that the triplet state is lower in energy than the possible singlet or open-shell singlet states, consistent with the observed EPR spectrum (Fig 3d). For both [5] ${ }^{2-}$ or [6] ${ }^{2-}$ calculations suggest that the triplet state is also lower in energy than the closed-shell singlet state (see SI). However, in the case of $[5]^{2-}$ the EPR spectrum (Fig $3 e$ ) shows features consistent with a triplet state and a signal that could be consistent with an open-shell singlet diradicaloid. The multireference perturbation theory calculations for $[5]^{2-}$ and $[6]^{2-}$ indicate small energy gaps, of $6.1 \times 10^{-4}$ a.u. and $1.5 \times 10^{-4}$ a.u. respectively, between triplet state and open-shell singlet diradicaloid states. We propose that both states are apparent in the frozen solution EPR spectrum of $[5]^{2-}$, whereas for [6] ${ }^{2-}$ the frozen solution EPR spectrum suggests that the triplet state is favoured. Although the calculations suggest that the open-shell singlet is favoured for $[6]^{2-}$ the calculated energy gap to triplet is smaller in this case than for [5] ${ }^{2-}$ and we cannot rule out the presence of open-shell singlet state in the EPR spectrum. It is clear that the nature of the radicals for both $[5]^{2-}$ and $[6]^{2-}$ are finally balanced between open-shell singlet and triplet states and that this balance is probably affected by subtle changes in conditions.

a)

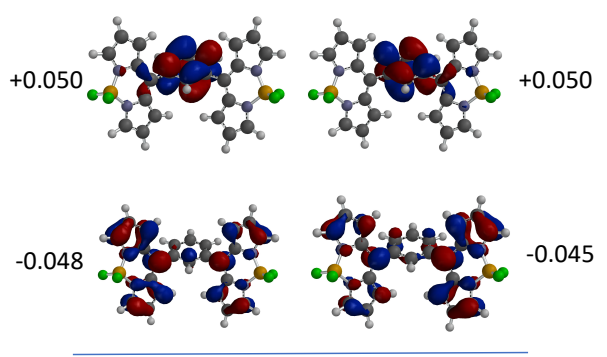

$-0.300$

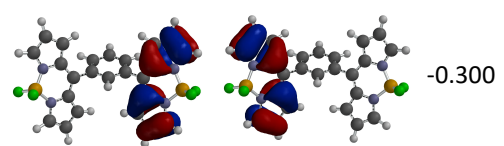

$-0.357$

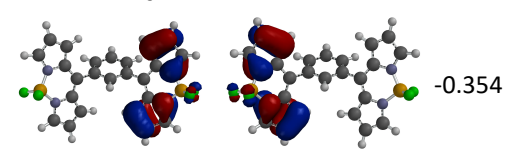

b)

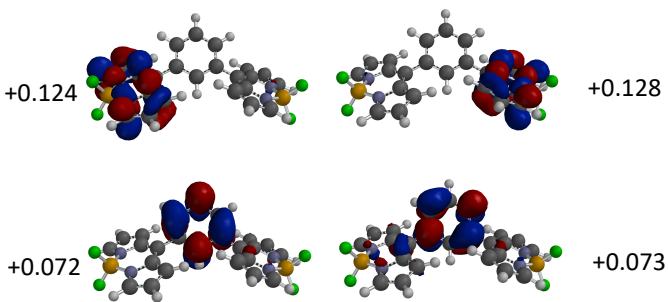

$-0.207$
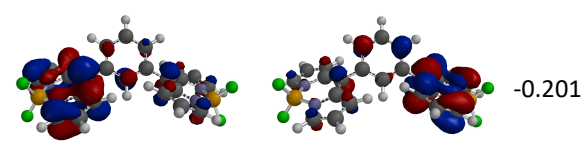

$-0.296$

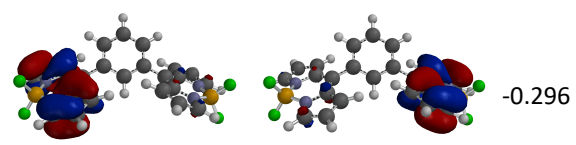

Fig. 5. DFT computed MO diagram for a) $\mathbf{2}$ in neutral ground state; b) the triplet form of [2] ${ }^{2-}$ with the energies of the molecular orbitals in atomic units. The line represents boundary 
between occupied and unoccupied orbitals. For the triplet state of $[2]^{2-}$ the $\alpha$ spin orbital energies are shown.

Spectroelectrochemical measurements were employed to further investigate the behaviour of 1-6 by UV-visible spectroscopy, with spectra recorded whilst reducing the sample in $\mathrm{CH}_{2} \mathrm{Cl}_{2}$ at $243 \mathrm{~K}$, with $\left[{ }^{n} \mathrm{Bu}_{4} \mathrm{~N}\right]\left[\mathrm{BF}_{4}\right]$ as supporting electrolyte. All compounds exhibited a sharp absorption band at approximately $500 \mathrm{~nm}$ with a large extinction coefficient of 90,000-120,000 dm $\mathrm{mol}^{-1} \mathrm{~cm}^{-1}$, featuring a shoulder to higher energy (Fig 6, Table S1) and a broader absorption band at approximately $330-400 \mathrm{~nm}$, with a smaller extinction coefficient of $24,000-33,000 \mathrm{dm}^{3} \mathrm{~mol}^{-1} \mathrm{~cm}^{-1}$. These bands are consistent with other BODIPY species, the most intense absorption band being associated with $\mathrm{S}_{0} \rightarrow \mathrm{S}_{1}$ transitions and the lower intensity process being due to $S_{0} \rightarrow S_{2}$ transitions. ${ }^{22}$ Electrochemical reduction with concurrent monitoring of the UV-vis spectrum of each compound revealed some clear trends with three distinct sets of features observed across the range of radicals generated. Each dianion, or trianion, exhibits two bands at $c a .550$ and $500 \mathrm{~nm}$. In all cases these two bands are clearly defined with the exception of [1] $]^{2-}$ for which the bands are not clearly resolved. These features are also observed for the monoradical anion of $\mathrm{N}, \mathrm{N}^{\prime}$-difluoroboryl-5-(phenyl)dipyrrin indicating a common chromophore is generated across the series, except for $[\mathbf{1}]^{2-2}{ }^{21} \mathrm{~A}$ further higher energy feature is seen for all radicals at $c a$. 300-400nm although for $[1]^{2-}$ this feature is less well defined and is lower in intensity (Fig 6a, Table S1). In addition, dianions [1] ${ }^{2-},[5]^{2-}$, and [6] ${ }^{2-}$ also display a low energy feature between 600 and 700nm (Fig 6a, e, f). It should be noted that in each case the parent, neutral, species was not fully recovered upon reoxidation of the samples indicating a small degree of decomposition of the reduced radical species under the conditions of the experiment.

Thus, the UV-visible spectra of $[1]^{2-},[5]^{2-}$ and $[6]^{2-}$ are significantly different to those observed for $[2]^{2-},[3]^{3-}$ or $[4]^{2-}$ with a relatively intense band observed at $>600 \mathrm{~nm}$. The nature of this lower energy band for $[\mathbf{1}]^{2-},[5]^{2-}$ and $[\mathbf{6}]^{2-}$ was probed further using DFT/TDDFT calculations ( $\omega$ B97M-V /6-311G*) (see Fig S17 for calculated spectra). The calculated UVvisible spectra of $[2]^{2-}$ and $[4]^{2-}$ are consistent with a triplet state assignment, confirmed by the EPR spectroscopic results and the calculated spectrum for [3] ${ }^{3-}$ predicts no low energy transitions corresponding to the trianion quartet state (Fig S17) in good agreement with experimental data. The experimental spectra for [5] ${ }^{2-}$ and $[6]^{2-}$ exhibit low energy bands which are best explained by a combination of both triplet and open-shell singlet states (Fig S18) as observed for [5] ${ }^{2-}$ in the EPR spectrum. Although the open-shell singlet state of [6] ${ }^{2-}$ is not apparent in the X-band EPR spectrum recorded as a frozen solution at $77 \mathrm{~K}$ we note that the UV-visible spectra were recorded at $243 \mathrm{~K}$, a temperature difference that may perturb the equilibrium between the triplet and open-shell singlet states.

The UV-visible spectrum of $[\mathbf{1}]^{2-}$ is quite different from those observed for the other species studied herein and TDDFT calculations appear to overestimate the intensities of transitions $<400 \mathrm{~nm}$. As the EPR spectrum for this species gave no evidence for either a triplet or open-shell singlet state and is strongly indicative of a closed-shell singlet we focussed our efforts on this system. TDDFT calculations of the closed-shell singlet state (Fig S17) indicated a low energy band at ca. $900 \mathrm{~nm}$ which was not observed experimentally, however, enforcing planarity between the dipyrrin moieties and the linking phenyl ring increased the energy of this band to ca. 750nm (Fig S19), that is in better agreement with the observed experimental spectrum. However, bands to high energy were not modelled well and in particular the lower experimental intensity of these features. Despite the difficulties in modelling the spectrum 
observed for $[\mathbf{1}]^{2-}$, in general the calculations are consistent with the radical state observed by EPR spectroscopy (Table 1).

In this study we demonstrate that it is possible to link BODIPY moieties into dyads and a triad, each of which can each be reduced to form radicals with varying spin multiplicities including closed and open shell singlet, triplet and quartet species. The diradical species can be considered as BODIPY analogues of the classic Thiele and Chichibabin hydrocarbons, ${ }^{38}$ which exist either as a quinoidal or a biradical benzenoidal form, and the Schlenk diradical, which due to the 1,3-substitution at the phenyl linker is restricted to a biradical benzenoid configuration (see SI, Scheme S1). ${ }^{39}$ We show that the geometrical arrangement of the linked BODIPYs influences the magnetic behaviour of the reduced BODIPY anions containing multiple BODIPY centres. Significant differences in the electrochemical behaviour, paramagnetism and UV-visible spectra of the reduced dyads, or triad, are observed. Thus, whereas a single reduction process was observed for para-substituted BODIPYs $\mathbf{1}, \mathbf{5}$ and $\mathbf{6}$, two processes were observed for the meta-substituted $\mathbf{2}$, duryl-linked $\mathbf{4}$ and triad $\mathbf{3}$. The EPR spectra of the reduced forms reflect similar redox behaviour of dyads $\mathbf{2}$ and $\mathbf{4}$ and triad $\mathbf{3}$ with the radicals anions forming triplet $\left([2]^{2-},[4]^{2-}\right)$ or quartet states $\left([3]^{3-}\right)$ consistent with formalization as bi- (or tri-) radical benzenoids. Our experimental observations are supported by DFT calculations and multireference perturbation theory which reveal the preferential adoption of triplet, or quartet, states for these three compounds in contrast to $[1]^{2-},[5]^{2-}$ or $[6]^{2-}$. In contrast, EPR silent $[\mathbf{1}]^{2-}$ is consistent with its formalization as a diamagnetic quinoidal arrangement and this differs from the behaviour of its paramagnetic tetramethyl substituted phenyl-bridged analogue, $[4]^{2-}$. Adopting the quinoid/biradical benzenoid formalization this difference illustrates the effect of spacer orientation restricting conjugation between redox centres thus imposing a biradical benzenoidal configuration. The para-substituted phenyl $\left([1]^{2-}\right)$, biphenyl $\left([5]^{2-}\right)$ and terphenyl $\left([6]^{2-}\right)$ bridged compounds are all capable of conjugation (see $\mathrm{SI}$ ) that extends between BODIPY centres and all exhibit bands lower in energy than 550 $\mathrm{nm}$ in their UV-Vis spectra which would be consistent this as an interpretation $11,35,38,40$ and would imply a preference for the quinodal form in this series (see $\mathrm{SI}$ ). This interpretation is reasonable for EPR silent $[\mathbf{1}]^{2-}$ but both $[5]^{2-}$ and $[6]^{2-}$ are paramagnetic implying a biradical benzenoidal configuration is preferred, either as a triplet or open shell singlet state, as a result of the larger separation between the reduced BODIPY moieties. Hence by increasing the distance between these BODIPY centres both triplet, observed for $[5]^{2-}$ and $[6]^{2-}$, and openshell singlet states, observed for [5] $]^{2-}$ play a significant role in the magnetic behaviour.

Our strategy gives insight into how BODIPY dyads and triads can be reduced to afford radical anions with varying multiplicities and how both molecular geometry and the number of reduced BODIPY groups can lead to systems with high multiplicity. Thus, our study reveals BODIPY species as a fascinating alternative for the formation of radicaloids with controllable spin states. 

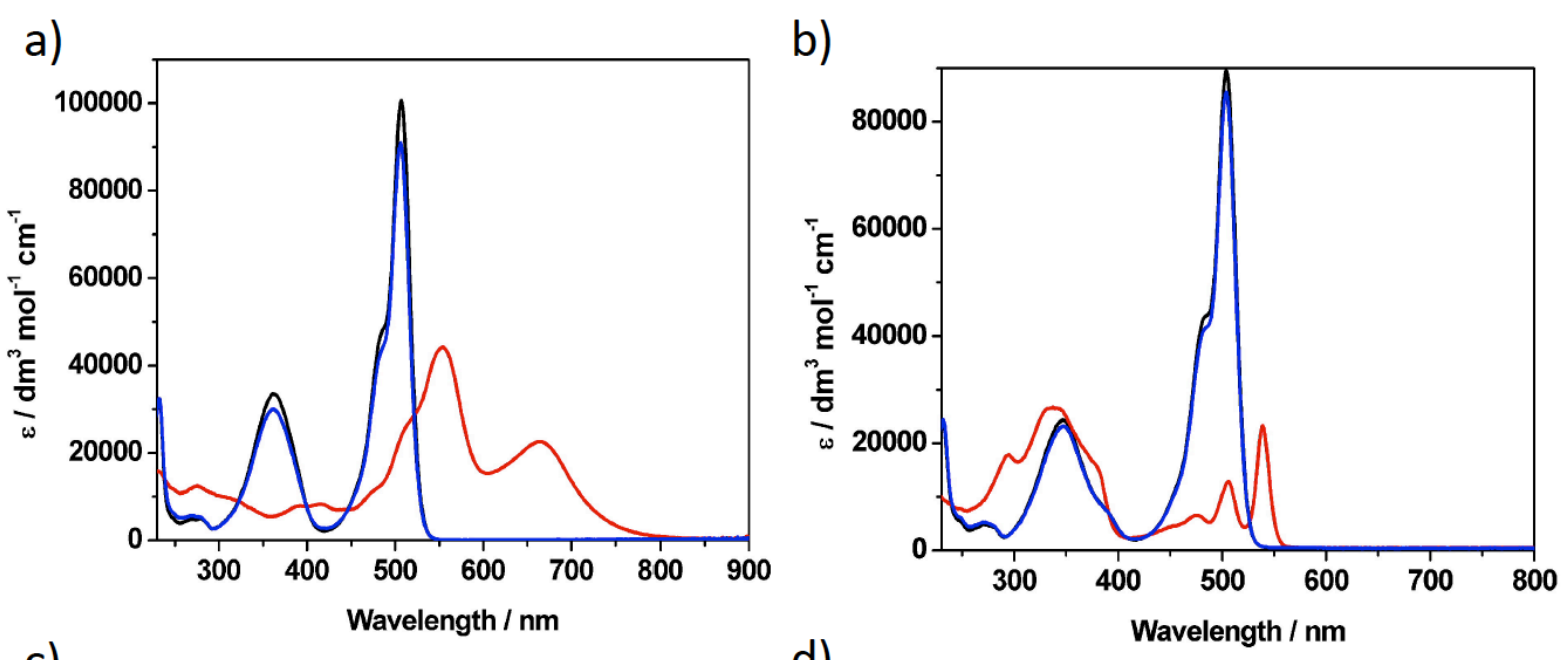

C)
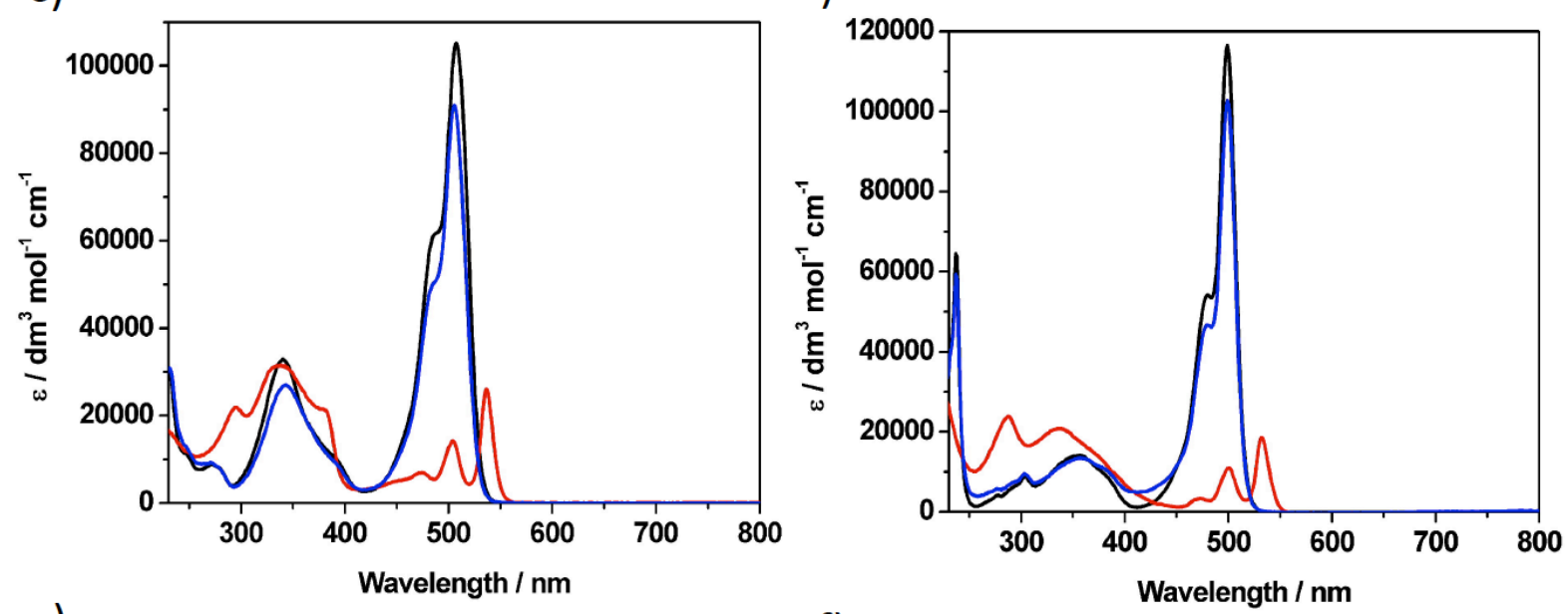

e)
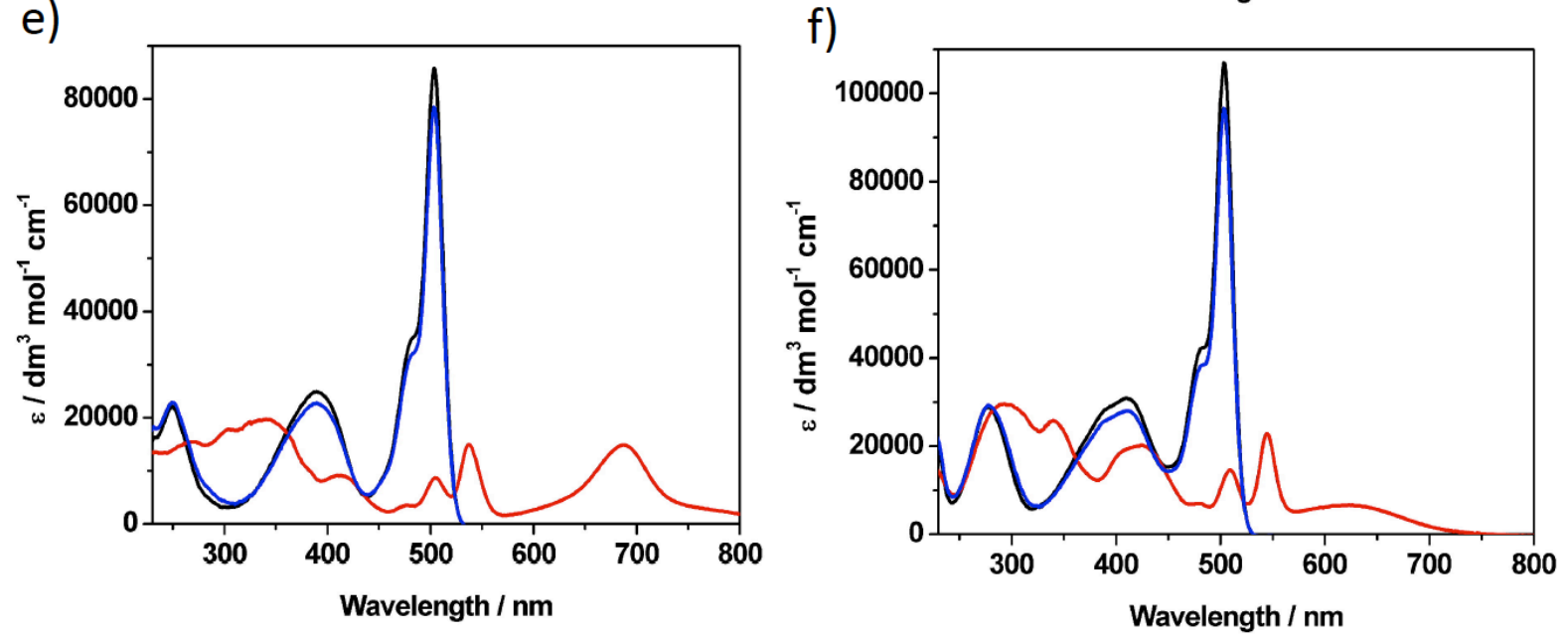

Fig. 6. UV-visible spectra for a) $1 /[1]^{2-}$; b) $2 /[2]^{2-}$; c) $3 /[3]^{3-}$; d) $4 /[4]^{2-}$; e) $5 /[5]^{2-}$; f) $6 /[6]^{2-}$

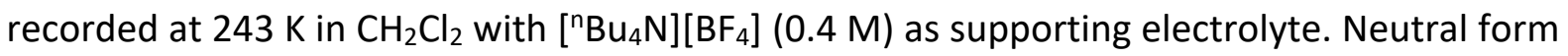
- black; reduced radical dianion (or trianion) - red; spectrum of reoxidised neutral - blue. Note loss of intensity for reoxidsed samples indicating degradation of reduced sample.

Supporting Information Available: Full experimental details and additional figures for electrochemistry, spectroelectrochemistry, EPR spectra, DFT and multireference perturbation 
theory calculations. Details of structural refinement and CIF file for $\mathbf{1}, \mathbf{2}$ and $\mathbf{3}$. CCDC 19350571935059 contain the supplementary crystallographic data for this paper. These data can be obtained free of charge from the Cambridge Crystallographic Data Centre via www.ccdc.cam.ac.uk/data request/cif.

\section{Conflicts of interest}

There are no conflicts to declare.

\section{Author Information}

Corresponding Author

*E-mail: Neil.Champness@nottingham.ac.uk

\section{Acknowledgements}

We acknowledge financial support from the UK Engineering and Physical Sciences Research Council (EP/N033906/1) and the Industrialization of Science and Technology Project, China (2014A35001-2) for financial support.

\section{References}

1. I. Ratera, J. Veciana, Chem. Soc. Rev., 2012, 41, 303-349.

2. J. Hankache, O. S. Wenger, Chem. Rev., 2011, 111, 5138-5178.

3. S. Kumar, Y. Kumar, S. K. Keshri, P. Mukhopadhyay, Magnetochemistry, 2016, 2, 42.

4. K. Okada, T. Imakura, M. Oda, H. Murai, J. Am. Chem. Soc. 1996, 118, 3047-3048.

5. A. Rajca, Y. Wang, M. Boska, J. T. Paletta, A. Olankitwanit, M. A. Swanson, D. G. Mitchell, S. S. Eaton, G. R. Eaton, S. Rajca, J. Am. Chem. Soc., 2012, 134, 15724-15727.

6. N. M. Gallagher, J. J. Bauer, M. Pink, S. Rajca, A. Rajca, J. Am. Chem. Soc., 2016, 138, 9377-9380.

7. A. Rajca, A. Olankitwanit, Y. Wang, P. J. Boratyński, M. Pink, S. Rajca, J. Am. Chem. Soc., 2013, 135, 18205-18215.

8. K. Cai, M. C. Lipke, Z. Liu, J. Nelson, T. Cheng, Y. Shi, C. Cheng, D. Shen, J-M. Han, S. Vemuri, Y. Feng, C. L. Stern, W. A. Goddard III, M. R. Wasielewski, J. F. Stoddart, Nat. Commun., 2018, 9, 5275.

9. X. Yu, A. Mailman, K. Lekin, A. Assoud, C. M. Robertson, B. C. Noll, C. F. Campana, J. A. K. Howard, P. A. Dube, R. T. Oakley, J. Am. Chem. Soc. 2012, 134, 2264-2275.

10. B. D. Koivisto, A. S. Ichimura, R. McDonald, M. T. Lemaire, L. K. Thompson, R. G. Hicks, J. Am. Chem. Soc. 2006, 128, 690-691.

11. T. Y. Gopalakrishna, W. Zeng, X. Lu and J. Wu, Chem. Commun., 2018, 54, 2186-2199.

12. M. Winkler, W. Sander, Acc. Chem. Res., 2013, 47, 31-44.

13. M. Tanaka, K. Matsuda, T. Itoh, H. Iwamura, J. Am. Chem. Soc., 1998, 120, 7168-7173.

14. A. I. Krylov, J. Phys. Chem. A, 2005, 109, 10638-10645.

15. H. F. Bettinger, W. Sander, J. Am. Chem. Soc. 2003, 125, 9726-9733.

16. W. Sander, D. Grote, S. Kossmann, F. Neese, J. Am. Chem. Soc. 2008, 130, 4396-4403.

17. D. Grote, C. Finke, S. Kossmann, F. Neese, W. Sander, Chem. Eur. J. 2010, 16, 44964506.

18. A. Rajca, A. Olankitwanit, Y. Wang, P. J. Boratyński, M. Pink, S. Rajca, J. Am. Chem. Soc., 2013, 135, 18205-18215. 
19. Y. Zheng, M-S. Miao, G. Dantelle, N. D. Eisenmenger, G. Wu, I. Yavuz, M. L. Chabinyc, K. N. Houk, F. Wudl, Adv. Mater., 2015, 27, 1718-1723.

20. C. D. Martin, M. Soleilhavoup, G. Bertrand, Chem. Sci., 2013, 4, 3020-3030.

21. M. E. Itkis, X. Chi, A. W. Cordes, R. C. Haddon, Science, 2002, 296, 1443-1445.

22. V.J. Richards, A.L. Gower, J.E.H.B. Smith, E.S. Davies, D. Lahaye, A.G. Slater (neé Phillips), W. Lewis, A.J. Blake, N.R. Champness, D.L. Kays, Chem. Commun., 2012, 48, 1751-1753.

23. A. Loudet, K. Burgess, Chem. Rev., 2007, 107, 4891-4932.

24. T. Kowada, H. Maeda and K. Kikuchi, Chem. Soc. Rev., 2015, 44, 4953-4972.

25. C. S. Kue, S. Y. Ng, S. H. Voon, A. Kamkaew, L. Y. Chung, L. V. Kiew and H. B. Lee, Photochem. Photobiol. Sci., 2018, 17, 1691-1708

26. P.E. Kesavan, S. Das, M.Y. Lone, P.C. Jha, S. Moric, I. Gupta, Dalton Trans., 2015, 44, 17209-17221.

27. M.T. Whited, N.M. Patel, S.T. Roberts, K. Allen, P.I. Djurovich, S.E. Bradforth, M.E. Thompson, Chem. Commun., 2012, 48, 284-286; A. Coskun, E.U. Akkaya, J. Am. Chem. Soc., 2006, 128, 14474-14475; G. Barin, M. D. Yilmaz, E. U. Akkaya, Tetrahedron Lett., 2009, 50, 1738-1740; R. Matsuoka, T. Nabeshima, Front. Chem., 2018, 6, 349.

28. T. Li, W. Gu, C. Yu, X. Lv, H. Wang, E. Hao, L. Jiao, Chin. J. Chem. 2016, 34, 989-996; L. Porrès, O. Mongin, M. Blanchard-Desce, Tetrahedron Lett., 2006, 47, 1913-1917.

29. C.-H. Lee, J. S. Lindsey, Tetrahedron, 1994, 50, 11427-11440

30. A. C. Benniston, S. Clift, A. Harriman, J. Mol. Struct., 2011, 985, 346-354

31. P. Saville, Polypyrrole Formation and Use, 2005, Defence R\&D Canada - Atlantic.

32. A. J. Bard and L. R. Faulkner, Electrochemical Methods Fundamentals and Applications, Second Edition, Wiley, 505-509, 2001.

33. It is noted that the couple exhibits scan rate dependence and, at slower scan rates $\left(0.20 \mathrm{Vs}^{-1}\right)$ the peak separation drops to $0.05 \mathrm{~V}$, significantly less than that of the ferrocene couple under identical conditions $(0.07 \mathrm{~V})$. Such behaviour in electrochemical EE-type mechanisms occurs when there are attractive interactions on addition of a second electron. ${ }^{31}$

34. J. Vecina, C. Rovira, M. I. Crespo, O. Armet, V. M. Domingo and F. Palacio, J. Am. Chem. Soc., 1991, 113, 2552-2561; A. Olankitwanit, M. Pink, S. Rajca and A. Rajca, J. Am. Chem. Soc., 2014, 136, 14277-14288; D. A. Shultz, A. K. Boal and G. T. Farmer, J. Org. Chem., 1998, 63, 9462-9469;

35. A. Rebmann, J. Zhou, P. Schuler, A. Rieker and H. B. Stegmann, J. Chem. Soc, Perkin Trans. 2, 1997, 1615-1617.

36. A. Vysniauskas, I. López-Duarte, N. Duchemin, T-T. Vu, Y. Wu, E. M. Budynina, Y. A. Volkova, E. Penã Cabrera, D. E. Ramírez-Ornelas and M. K. Kuimova, Phys. Chem. Chem. Phys., 2017, 19, 25252-25259.

37. H. Zhang, H. Phan, T. S. Herng, T. Y. Gopalakrishna, W. Zeng, J. Ding and J. Wu, Angew. Chem., Int. Ed., 2017, 56, 13484-13488.

38. G. Tan and X. Wang, Acc. Chem. Res., 2017, 50, 1997-2006.

39. A. Rajca and S. Rajca, J. Chem. Soc., Perkin Trans. 2, 1998, 1077

40. J.P. Telo and M.F.N.N. Carvalho, J. Photochem. Photobiol. A, 2019, 376, 140-145. 